\title{
Experimental Investigation of Mixed Convection on a Rotating Circular Cylinder in a Cavity Filled With Nanofluid and Porous Media
}

\author{
Ahmed Dhafer Abdulsahib ${ }^{a}$, Khaled Al-Farhany ${ }^{a *}$ \\ ${ }^{a}$ Department of Mechanical Engineering, University of Al-Qadisiyah, Ad-Diwaniyah (58001), Iraq
}

\section{A R T I C LE INFO}

Article history:

Received 03 May 2020

Received in revised form 16 June 2020

Accepted 26 June 2020

\section{Keywords:}

Nanofluid

Porous media

Two layers

Circular cylinder

Rotating cylinder

Mixed convection

\begin{abstract}
A B S T R ACT
The present study, experimentally investigated the mixed convection in a square enclosure partitioned in two layers. The experiments were performed with $\mathrm{Al}_{2} \mathrm{O}_{3}$-water nanofluid (upper layer) and superposed porous medium (lower layer) with an adiabatic rotating cylinder at the center of the cavity. The boundary conditions of the experimental study were; the upper and lower walls were assumed adiabatic, the right wall was heated, and the left wall was cooled. Experimentally, $15 \mathrm{~K}$-type thermocouples and thermal imaging camera were employed to measure the temperatures distribution inside the cavity when the concentration of nanoparticles $(\phi=0.06)$, the temperature difference $(\Delta \mathrm{T})$ between the cold and hot walls was $(6,8$, and 10$)$ ${ }^{\circ} \mathrm{C}$, and angular rotational velocity $(-50,-25,0,25$, and 50$) \mathrm{rpm}$. The results of experimental data showed that in general, the distribution of temperatures was very well along the upper half of the enclosure, while in the lower half the temperature distribution was confined near the hot wall region. When the circular cylinder rotates in counter-clockwise, it noted that the effect of speed is evident in the downside of the cylinder, while the temperature distribution in the left upper part of the enclosure decreasing. When the circular cylinder rotates in the clockwise direction, the results showed that the effect of cylinder rotation was around cylinder only. Moreover, the results demonstrated that the increasing temperature difference leads to a noticeable increment in the intensity of the flow.
\end{abstract}

\section{Introduction}

The studies of enhancement convection heat transfer inside the cavity, that has received the attention of researchers because of its multiple engineering applications such as solar collectors, nuclear reactors, electronic equipment cooling, and heat exchangers [1-3]. By adding solid nanoparticle to base fluid, the enclosure is developed by repair its geometry, adding fins or baffles and inserting objects inside the enclosure In fixed case or rotating case, etc. [4-6].

* Corresponding author.

E-mail address: khaled.alfarhany@qu.edu.iq (Khaled Al-Farhany)
For controlling and enhancement of the convection heat transfer, a liddriven wall is used [7-14] and a rotating cylinder used inside the enclosures [15]. When using Rayleigh number 1-1400 show the influence of the rotating cylinder to increasing thermal transfer by Lewis [16]. Wu-Shung et al. [17] find the contribution of the counter-clockwise rotation cylinder in the heat transfer $60 \%$ when used $\mathrm{Gr} / \mathrm{Re}^{2}$ is $10^{3}$. It was compared with previous studies and the effect of the rotating cylinder on the heat transfer and flow patterns by Yang et al. [18]. Natural, mixed, and forced convection 
studied by Misirlioglu and Aydin [19] found when the cylinder rotates at a high speed, the maximum heat transfer is achieved. Costa et al. [20] appear the important effect of cylinder thermal properties inside enclosures on heat transfer. Hussain et al. [21] explain the effect of Richardson number and Reynolds number on average Nusselt number, heat transfer, and the field of fluid, as well as the effect of changing the cylinder location on the convection heat transfer. Roslan et al. [22] using several types of nanofluids with different concentrations and showed that rising the heat transfer when increasing the nanofluid concentrations, as well as the effect of heat transfer when the cylinder rotates in clockwise and counter-clockwise direction, The high heat transfer was found when using nanoparticles with the high concentration and good conductivity values when the rotating cylinder was in the centre of the cavity. By using a rotating cylinder inside horizontal annulus Matin et al. [23] explained the effect of Reynolds number on the average Nusselt number, as well as the effect of the location rotating cylinder. Mixed convection MHD was studied by Selimefendigil et al. [24] and show an increase in entropy and heat transfer with increasing concentration of nanoparticle. Numerical study of a hot rotating cylinder inside a cooled walls square enclosure by Liao et al. [25] and find average heat transfer at the rotating cylinder and the walls of the enclosure. Shih et al. [26] show that the triangular enclosure has been shown the greatest ability to dissipate thermal energy compared to the circular enclosure. By using two rotating cylinders inside a three-dimensional cavity, using nanofluid with different concentrations study by Selimefendigil et al. [27]. To enhance the heat transfer at two layers, a rotating cylinder has been added. Mixed convection that studied by Selimefendigil et al. [15] two layers nanofluid and porous medium inside the cavity and show the enhancement of Nusselt number when the cylinder rotates. Also two layers study by Hussein et al. [28]. The experimental study within nanofluid enclosure investigations by researchers[29, 30]. Solomon et al. [31] experimental study on the influence of the aspect ratio of square cavity on natural convection heat transfer with $\mathrm{Al}_{2} \mathrm{O}_{3}$ /Water nanofluids. Wen and Ding [32] showed a reduced mean size of accumulation by applying a highshear homogenizer. Joshi and Pattamatta [33] found that the lower value of Nusselt number when used (MWCNT/water), while get the higher value in (graphene/water) if compared with (alumina /Water). Khalili et al. [34] showed that the average size of the nanoparticles at the cold side wall was $3.10 \%$ higher than that along the hot wall.

Recently, many studies had been done with conviction/mixed heat transfer. A few studies have been done regarding using two layers (nanofluids and porous medium layers) with a rotating cylinder at different angular rotational velocity.

In this study, a mixed convection on the square cavity having two different horizontal layers with a rotating cylinder (clockwise and counterclockwise) is investigated experimentally. The upper and lower walls of the cavity are insulated thermally while the right wall is heated and the left wall is kept at low temperature.

\section{The Experimental Work}

This section describes the experimental rig design and its main components. It also shows the preparation of nanofluid and the type of porous medium used in the present work.

\subsection{Experimental Rig}

This section shows the description of the main units of the experimental setup. Fig. 1 and Fig. 2 illustrate the schematic diagram of the experimental setup and the schematic diagram of the experimental set-up respectively. This includes the enclosure, the stepper motor used to rotate the cylinder,

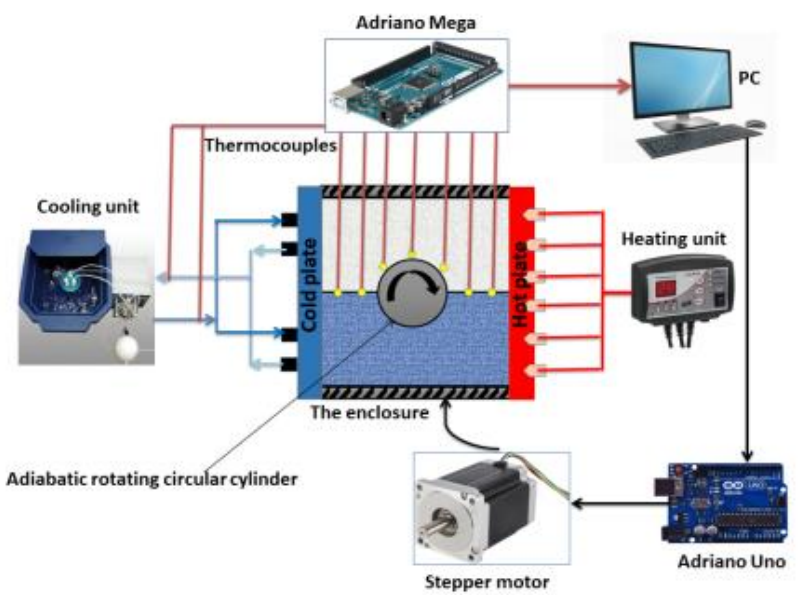

the heating unit (consists of a heater, a controller, and a DC power supply), the cooling unit (water bath at a constant temperature) and the measurement system (thermal camera, data logger tool, thermocouples, and PC).

Figure 1. Schematic diagram of the experimental setup

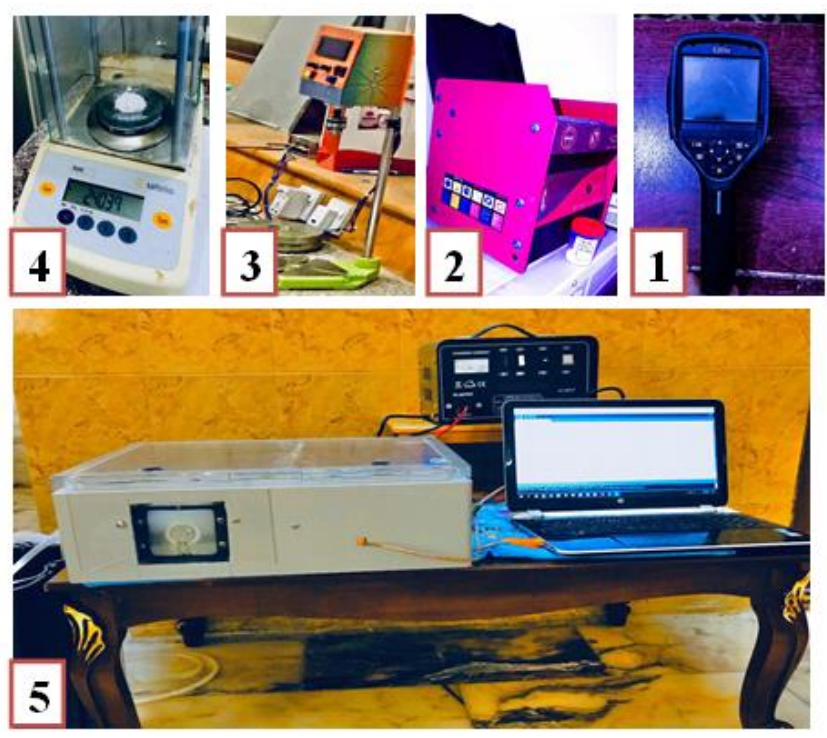

Figure 2. Experimental set-up apparatus: (1) FLIR E30bx Thermal Imaging Camera, (2) Ultrasonic waves apparatus, (3) Mechanical stirrer, (4) Sensitive balance, (5) Data logger, PC, and Test Section. 


\subsection{Test Section}

Fig. 3 shows the test rig which is a square enclosure. The top, bottom, front and back walls are made of Perspex glass having a thickness of 0.5 $\mathrm{cm}$. The rotating circular cylinder made of PVC in $3 \mathrm{~cm}$ diameter at the center of the enclosure with thermal properties shown in Table 1. The right wall is made of the aluminum plate that heated by an electric heater to control the temperature. The left wall of the enclosure was designed from an aluminum heat exchanger to control the temperature by passing the water through it. Stepper motor was used to obtain rotate the internal cylinder of the enclosure at different rotational speeds. Three thermocouples were installed at each right and left walls to measure temperatures, and eight thermocouples were installed in the middle section of the cavity for measuring the temperature distribution inside the enclosure as shown in Fig 4. These thermocouples are programmed by using Arduino (MEGA) type with programming code.
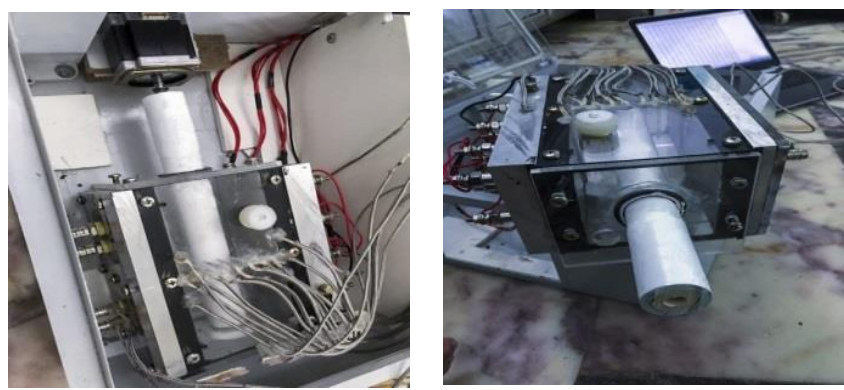

Figure 3. Enclosure assembly.

Table 1 The properties of Perspex glass [35]

\begin{tabular}{llc}
\hline Physical properties & Density & $\begin{array}{l}\text { Thermal expansion } \\
\text { coefficient }\end{array}$ \\
\hline Perspex glass & $(1.17-1.2)\left(\mathrm{g} / \mathrm{cm}^{2}\right)$ & $(5-10) \times 10^{5}\left(\mathrm{~K}^{-1}\right)$ \\
\hline
\end{tabular}

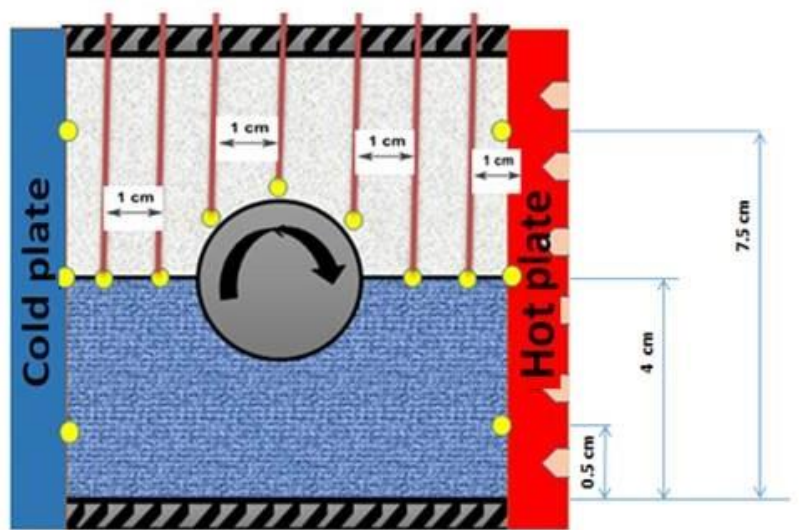

Figure 4. Thermocouples location inside the enclosure

\subsection{Formulation and characterization of porous media}

The porous medium is an object consisting of a fixed solid part called a solid matrix that contains empty spaces that can be filled with fluid. The solid matrix that used in present work made of glass bead with diameter 3 $\mathrm{mm}$ as shown in Fig. 5. The properties of the glass bead sphere take from Table 2. The porosity $(\varepsilon)$ and permeability $(\mathrm{K})$ of the porous medium are computed from Eqs. 1 and 2 respectively.

$$
\begin{aligned}
& \varepsilon=\frac{V_{\text {void }}}{V_{\text {solid }}}=1-\frac{V_{\text {solid }}}{V_{\text {total }}} \\
& K=\frac{d_{p}^{2} \varepsilon^{3}}{150(1-\varepsilon)^{2}}
\end{aligned}
$$

- $\quad$ The mean value of the calculated porosity values for glass bead (3) mm diameter $\varepsilon=0.37$.

- The average value of the permeability for glass bead (3) $\mathrm{mm}$ in diameter $\left(\mathrm{K}=7.65 \times 10^{-9} \mathrm{~m}^{2}\right)$.
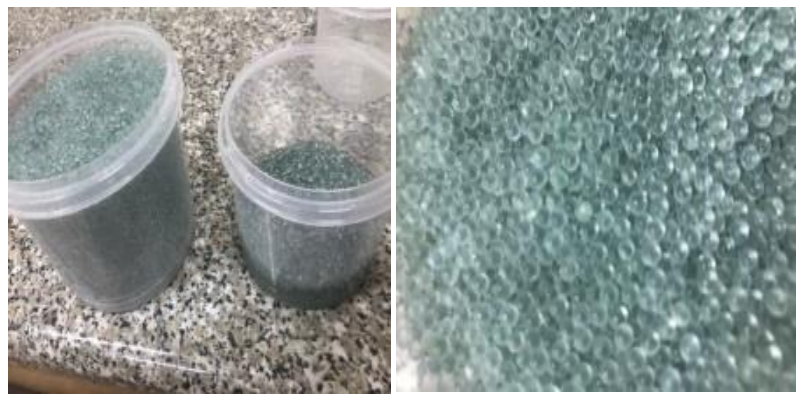

Figure 5. The porous media used in this work.

Table 2 Thermo-physical properties of some glass [36].

\begin{tabular}{lccc}
\hline Material & $\boldsymbol{\rho}_{\mathbf{s}}\left(\boldsymbol{k g} / \boldsymbol{m}^{\mathbf{3}}\right)$ & $\boldsymbol{C p}_{\boldsymbol{s}}(\boldsymbol{P a} . \boldsymbol{s})$ & $\boldsymbol{K}_{\boldsymbol{s}}(\boldsymbol{W} / \boldsymbol{m} . \boldsymbol{k})$ \\
\hline Glass bead & 2507 & 670 & 0.78 \\
Glass window & 2800 & 800 & 0.81 \\
Soda-lime & 2500 & 750 & 1.4 \\
Glass window & 2700 & 780 & 0.78 \\
\hline
\end{tabular}

\subsection{Preparation of nanofluid}

In the current work, $\mathrm{Al}_{2} \mathrm{O}_{3}$ nanoparticles are used having an average size between $40 \mathrm{~nm}$ and $80 \mathrm{~nm}$ and purity equal to $99.99 \%$.

The preparation of the nanofluid requires several processes that are summarized in the following points: 
1- To calculate the Nano-powder weight for all concentrations using Eq. 3 :

$$
\phi \%=\frac{\left(m_{\mathrm{p}} / \rho_{p}\right)}{\left(m_{\mathrm{p}} / \rho_{p}\right)+\left(m_{\mathrm{bf}} / \rho_{b f}\right)}
$$

2- The sensitive balance that has been used to get the required quantity of mass as shown in Table 3.

3- To obtain an efficient and uniform distribution of nanoparticles as well as a steady mixture by using ultrasound waves (sonication bath for 4 hours).

4- Use Arabic Gum [37] with $0.25 \%$ of base fluid like surfactants for improving the stability of the nanofluid.

5- To ensure the homogeneity of the mixture and attain the required volume fraction of the nanoparticle using the mechanical stirrer for 1 hour.

Table 3. Mass of $\mathrm{Al}_{2} \mathrm{O}_{3}$ nano-powder for different concentrations

\begin{tabular}{ll}
\hline $\begin{array}{l}\text { The concentration of } \\
\text { Nanoparticles \% }\end{array}$ & Mass of $\mathrm{Al}_{2} \mathrm{O}_{3}$ Nanoparticles $(\mathrm{g})$ \\
\hline 0.02 & 0.79415 \\
0.04 & 1.588635 \\
0.06 & 2.38343 \\
\hline
\end{tabular}

Fig. 6 shows how the prepared nanofluid, using the above points, is used in the test rig.

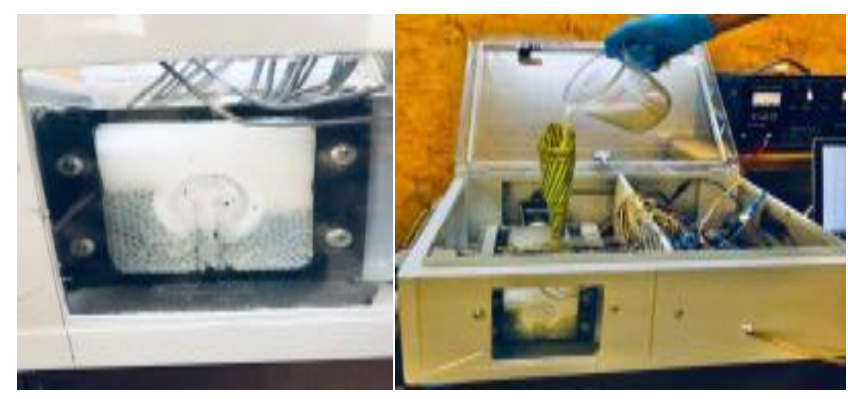

Figure 6. Nanofluid in the testing device

\subsection{Measuring the thermo-physical properties of the nanofluid}

In heat transfer, the thermophysical properties of the nanofluid play a significant role. The density, effective viscosity, and thermal conductivity of the nanofluid are computed from Eqs. 4 to 6. These parameters were introduced in the study achieved by Pak and Cho [38] and listed in Table4.

$$
\rho_{n f}=(1-\Phi) \rho_{f}+\Phi \rho_{n p}
$$

The dynamic viscosity is fined by using (Brinkman model):

$$
\mu_{n f}=\frac{\mu_{f}}{(1-\Phi)^{2.5}}
$$

The thermal conductivity is fined by using (Maxwell correlation):

$$
K_{n f}=K_{f} \frac{\left(K_{n p}+2 K_{f}\right)-2 \Phi\left(K_{f}-K_{n p}\right)}{\left(K_{n p}+2 K_{f}\right)+\Phi\left(K_{f}-K_{n p}\right)}
$$

Table 4. The variation of the nanofluid $\left(\mathrm{Al}_{2} \mathrm{O}_{3}\right.$ water) thermo-physical properties with the nanoparticles solid volume fraction.

\begin{tabular}{lllll}
\hline No & Sample & $\rho_{\text {nf }}\left(\mathbf{k g} / \mathbf{m}^{3}\right)$ & $\mu_{\text {nf }}($ Pa.s $)$ & $\boldsymbol{K}_{\boldsymbol{n} \boldsymbol{f}}(\boldsymbol{W} / \mathbf{m} . \boldsymbol{k})$ \\
\hline 1 & Base fluid & 997.07 & 0.001003 & 0.61300 \\
2 & $\Phi=0.06$ & 1175.5 & 0.0011708 & 0.72483 \\
\hline
\end{tabular}

\subsection{Experimental procedure and calculations}

In this work, 45 experiments were performed. These experiments are divided into groups using different parameters; the concentration of nanoparticles, the difference in temperature between the hot and cold walls, and the insulated cylinder angular velocity. Firstly, obtaining the thermophysical properties of the nanofluid at nanoparticle concentrations which are specified as $(0.02,0.04$, and 0.06). Secondly, the temperature differences between the cold and hot walls are taken equal to (6, 8, and10); which are not very large to avoid turbulent flow and also not very small to ensure a large flow in convective heat transfer. Third, after measuring all temperatures at steady state condition readings are taken inside the cavity for each insulated circular cylinder angular velocity of $-50,-25,0,25$, and 50 RPM. The temperature difference between the cavity and the cylinder wall was within the limits of the mixed convective heat transfer represented by Richardson's number $(0.1<\mathrm{Ri}<10)$.

This next section introduces the device initialization and verifies the accuracy of readings to carry out experiments in a wide range of different operational conditions.

\subsection{Device initialization}

There are several processes, represented the initialization of the device, that should be taken into account before conducting the experiments.

1. The enclosure was initially washed by distilled water to remove impurities and sediments.

2. Half of the cavity is filled with a porous matrix, after that, the nanofluid is added completely to prevent the formation of gas bubbles so that accuracies of the test results are obtained.

3. Heaters were placed at the hot wall including a controller and a suitable DC power supply; while a constant temperature water bath was used at the cold wall.

4. Temperature results are shown by displaying the data of thermocouples every 10 seconds and saved in the computer. 
5. After all, temperatures are measured in a stable condition, the insulated circular cylinder is rotated at the required speed.

6. After stabilization is reached when the temperature difference is less than $0.25^{\circ} \mathrm{C}$ in computer data for all points. Finally, a thermal image is taken by the thermal imaging camera (FLIR E30bx).

The experiments were conducted at the College of Engineering/ University of Al-Qadisiyah / Laboratories of the College of Mechanical Engineering.

\subsection{Achieve device readings}

To verify the device is working properly, the results of temperature distribution from a thermal camera were compared with eight thermocouples were installed in the middle section of the cavity. This includes two cases are shown in Fig. 7 for the temperature distribution at the horizontal axis (i.e. $x$-axis) at the enclosure middle line.

The comparison showed a very clear agreement for all cases and this is an important factor indicating the accuracy of the experimental part.

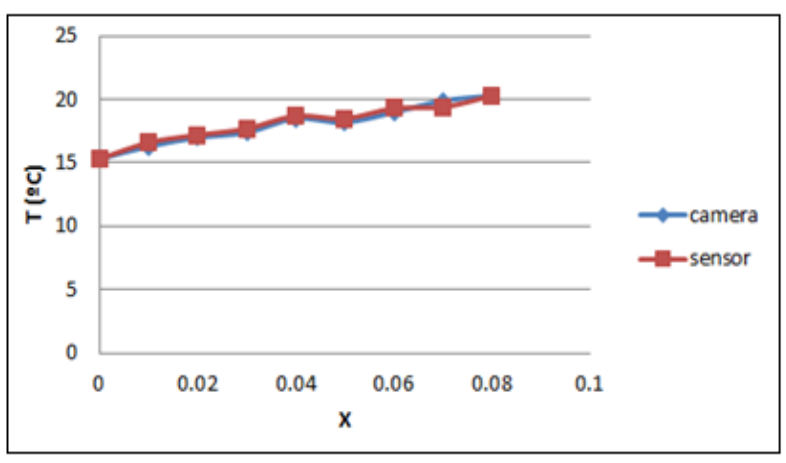

(a) For water at $(\mathrm{W}=\mathbf{0}, \Delta \mathrm{T}=\mathbf{5})$

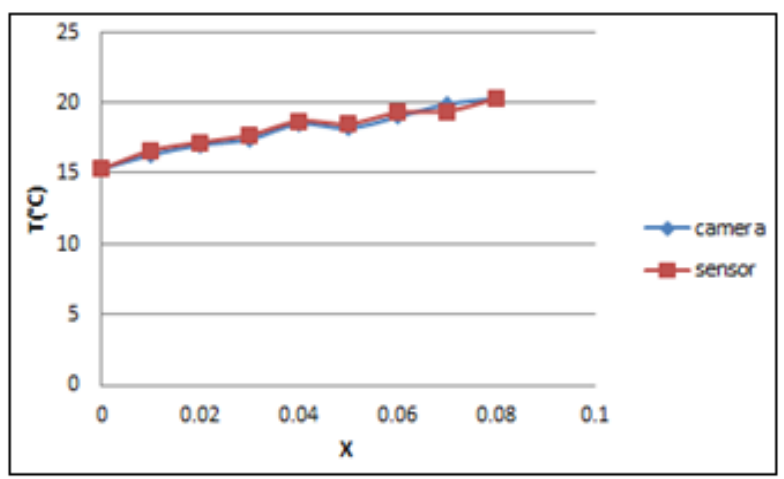

(b) For water at $(\mathrm{W}=-25, \Delta \mathrm{T}=8)$

Figure 7. Comparison of the results of the temperature distribution between the thermal camera and thermocouple in the $\mathrm{x}$-axis located at the enclosure middle-line.

\section{Results and Discussion}

In this case, the results are shown form the test rig to study mixed convection heat transfer. The enclosure is filled with a porous media to half, after that, the nanofluid is added to full the enclosure. The experimental results were taken by a thermal imaging camera and thermocouples to show the distribution of the temperature inside the cavity. In addition, the experimental results were compared, as mentioned represented by the thermal imaging camera and thermocouples.

The experiments are divided into four cases based on specific parameters as follows:

1. The concentration of nanoparticles $(0.02,0.04,0.06)$.

2. The temperature difference between the cold and hot walls $(6$, $8,10)$.

3. The angular rotational velocity of the insulated circular cylinder $(-50,-25,0,25,50)$ RPM

4. The inner circular cylinder radius $(\mathrm{r}=1.5 \mathrm{~cm})$.

\subsection{Effects of rotating circular cylinder with temperature difference $(\Delta T=6)$}

Fig.8 and Fig. 9 show the effect of angular rotational velocity on temperature distribution for $\mathrm{Al}_{2} \mathrm{O}_{3}$-water, $\phi=0.06, \Delta \mathrm{T}=6$. It can be seen from this figure the rotation of the cylinder is studied in five cases: two angular rotational velocities in counter-clockwise, two angular rotational velocities in a clockwise direction, and when the cylinder is stationary.

The figure shows that the distribution of temperatures is greater in the upper of the cavity, while it is confined near the hot wall in the lower half due to low permeability. The lower permeability impedes the fluid due to the use of low porosity of the porous matrix so that the conduction is dominant.

When $\omega=0 \mathrm{rpm}$ the fluid moves by natural convection showing different temperatures between the right hot vertical wall and the cold left wall depending on the buoyancy force. Therefore, the fluid flow moves from the right hot vertical wall towards the upper horizontal wall that is thermally insulated. Then the flow completes its cycle down affected by the cold left vertical wall reaching the insulated lower horizontal wall. It can be noticed that the presence of a thermally insulated cylinder disturbs the movement of the fluid, resulting in a decrease the distribution of temperature in the upper half to the right of the cylinder $\left(\mathrm{T}=19^{\circ} \mathrm{C}\right)$, as well as in the lower half the influence of the hot wall and the buoyancy force are weak at the left of the cylinder and $\left(\mathrm{T}=17.3^{\circ} \mathrm{C}\right)$.

When the circular cylinder rotates in a counter-clockwise at $(\omega=-25$ $\mathrm{rpm})$. Note the effect of speed is evident in the down of the cylinder where $\left(\mathrm{T}=19{ }^{\circ} \mathrm{C}\right.$ ), while the temperature distribution in the left upper part decreases. This is because the part of the fluid due to the rotation of the cylinder collides with the porous medium layer with little permeability to rise towards the isolated upper wall so it will prevent the buoyancy force. At $(\omega=-50 \mathrm{rpm})$ the influence of the rotational speed is more evident, where the same physical interpretation of $(\omega=-25 \mathrm{rpm})$, but the density of the heat distribution is greater. In this case, the temperature at the bottom of the 
cylinder increases from $\left(19^{\circ} \mathrm{C}\right.$ to $\left.20.5^{\circ} \mathrm{C}\right)$. Also, the distribution of heat increases relatively to include the area near the cold wall.

At $(\omega=25 \mathrm{rpm})$ the cylinder rotates clockwise direction where the influence of a rotating cylinder is opposite to the influence of buoyancy force. Notice that the effect of the cylinder to rotate the fluid is limited to the area near it where $\left(\mathrm{T}=18.7^{\circ} \mathrm{C}\right)$, while the effect of buoyancy force appears in other areas, especially in the upper part of the cavity where $\left(\mathrm{T}=20.2^{\circ} \mathrm{C}\right)$.

At $(\omega=50 \mathrm{rpm})$ the influence of the rotational speed of the cylinder is more influential than the buoyancy force inside most regions of the cavity, where the heat distribution density is greater. Therefore, the temperature at the bottom of the cylinder increases from $\left(19^{\circ} \mathrm{C}\right.$ to $\left.20.5^{\circ} \mathrm{C}\right)$. The heat distribution also increases relative to the area near the cold wall while it decreases at the upper right of the cavity. 


\subsection{Effects of rotating circular cylinder with temperature difference $(\Delta T=8)$}

Fig. 10 and Fig. 11 shows the effect of angular rotational velocity on temperature distribution for $\mathrm{Al}_{2} \mathrm{O}_{3}$-water, $\phi=0.06$, and $\Delta \mathrm{T}=8$. The same five cases, related to the rotation of the cylinder, examined at $\Delta \mathrm{T}=6$ are presented in this section for $\Delta \mathrm{T}=8$. The physical interpretation of the cylinder rotation is the same as in the previous case, but the temperature difference here is greater. It is interesting to a reminder that the increase in temperature difference, results in increasing the intensity of the flow, which in turn leads to an increase in the buoyancy force.

Considering mixed convection heat transfer inside an enclosure, there are two effects on the production of flow vortices, the first is the buoyancy force and the second is the sheer force of the rotational circular cylinder. Therefore, it can be seen that the influence of rotation of the cylinder is greater compared to the with the buoyant force if they are in the same direction while the effect of the cylinder is absent at lower speeds due to the high buoyancy force when there are different directions.

\subsection{Effects of rotating circular cylinder with temperature difference $(\Delta T=10)$}

Fig. 12 and Fig. 13 shows the effect of angular rotational velocity on temperature distribution for $\mathrm{Al}_{2} \mathrm{O}_{3}$-water, $\phi=0.06, \Delta \mathrm{T}=10$. The same five cases, related to the rotation of the cylinder, examined at $\Delta \mathrm{T}=8$ are presented in this section for $\Delta \mathrm{T}=10$. The physical interpretation of the cylinder rotation is the same as in the two previous cases, but the temperature difference here is greater. When $(\omega=0 \mathrm{rpm})$ the fluid moves by natural convection heat transfer at different temperatures between the right hot vertical wall and the cold left wall. It can be seen that due to the difference in high temperatures, the distribution of heat includes most regions of the cavity, and because of the accumulation of temperatures under the cylinder where it reaches $\mathrm{T}=22.3^{\circ} \mathrm{C}$.

In general, when the cylinder rotates, the heat transfer inside the cavity increases, and the reason is the influence of buoyancy and cylinder rotational force. This shows the importance of mixed convection in increasing heat transfer.

\section{Conclusions}

From the experiment results, some important conclusions can be presented as follows:

1. In general, it is observed that the distribution of temperatures that is greater in the upper half of the considered enclosure, while it is confined near the hot wall in the lower half due to low permeability that impedes the fluid because of using low porosity of porous matrix so the conduction is dominant.

2. When $(\omega=0 \mathrm{rpm})$, the fluid moves by natural convection heat transfer at different temperatures, the flow field inside the cavity is controlled only by the buoyancy force, and the presence of a thermally insulated cylinder disturbs the movement of the fluid.

3. When the circular cylinder rotates counter-clockwise, the effect of the speed is evident in the down of the cylinder, while the temperature distribution in the left upper part decreases because a part of the fluid due to the rotation of the cylinder collides with the porous medium layer with little permeability to rise towards the isolated upper wall so it prevents the buoyancy force.

4. When the circular cylinder rotates clockwise direction, the influence of a rotating cylinder is opposite to the influence of the buoyancy force. Notice that the effect of the cylinder to rotate the fluid is limited to the area near it, while the effect of buoyancy force appears in other areas, especially in the upper part of the cavity. When temperature difference increases, a noticeable increase in the intensity of the flow is observed due to the increase in temperature, which leads to increased buoyancy force. Therefore, in mixed convection heat transfer, there are two effects on the production of flow vortices inside the cavity; the first is the buoyancy force and the second is because of the sheer force that resulted from the rotating cylinder.

\section{REFERENCES}

[1] O. Mahian, L. Kolsi, M. Amani, P. Estellé, G. Ahmadi, C. Kleinstreuer, J.S. Marshall, M. Siavashi, R.A. Taylor, H. Niazmand, Recent advances in modeling and simulation of nanofluid flows-part I: fundamental and theory, Physics reports, (2018).

[2] O. Mahian, L. Kolsi, M. Amani, P. Estellé, G. Ahmadi, C. Kleinstreuer, J.S. Marshall, R.A. Taylor, E. Abu-Nada, S. Rashidi, Recent advances in modeling and simulation of nanofluid flows-part II: applications, Physics reports, (2018).

[3] Y.-C. Shih, J. Khodadadi, K.-H. Weng, A. Ahmed, Periodic fluid flow and heat transfer in a square cavity due to an insulated or isothermal rotating cylinder, Journal of Heat Transfer, 131(11) (2009) 111701.

[4] N.C. Markatos, K. Pericleous, Laminar and turbulent natural convection in an enclosed cavity, International Journal of Heat and Mass Transfer, 27(5) (1984) 755-772.

[5] A. Baïri, E. Zarco-Pernia, J.-M.G. De María, A review on natural convection in enclosures for engineering applications. The particular case of the parallelogrammic diode cavity, Applied Thermal Engineering, 63(1) (2014) 304-322.

[6] A.-M. Barik, K. Al-Farhany, Numerical Investigation of the Effect of Baffle Inclination Angle on Nanofluid Natural Convection Heat Transfer in A Square Enclosure, Al-Qadisiyah Journal for Engineering Sciences, 12(2) (2019) 61-71.

[7] A. Hussein, S. Hussain, Mixed Convection through a Lid-Driven AirFilled Square Cavity with a Hot Wavy Wall, International Journal of Mechanical and Materials Engineering, 5(2) (2010) 222-235.

[8] S. Saha, A. Hussein, G. Saha, S. Hussain, Mixed convection in a tilted lid-driven square enclosure with adiabatic cylinder at the center, International Journal of Heat and Technology, To be published, (2011).

[9] K.M. Gangawane, H.F. Oztop, N. Abu-Hamdeh, Mixed convection characteristic in a lid-driven cavity containing heated triangular block: Effect of location and size of block, International Journal of Heat and Mass Transfer, 124 (2018) 860-875. 
[10] T. Mahalakshmi, N. Nithyadevi, H.F. Oztop, N. Abu-Hamdeh, MHD mixed convective heat transfer in a lid-driven enclosure filled with Agwater nanofluid with center heater, International Journal of Mechanical Sciences, 142 (2018) 407-419.

[11] Q. Yu, H. Xu, S. Liao, Analysis of mixed convection flow in an inclined lid-driven enclosure with Buongiorno's nanofluid model, International Journal of Heat and Mass Transfer, 126 (2018) 221-236.

[12] M.S. Astanina, M.A. Sheremet, H.F. Oztop, N. Abu-Hamdeh, Mixed convection of $\mathrm{Al} 2 \mathrm{O} 3$-water nanofluid in a lid-driven cavity having two porous layers, International Journal of Heat and Mass Transfer, 118 (2018) 527-537.

[13] A.J. Chamkha, M.A. Ismael, Magnetic field effect on mixed convection in lid-driven trapezoidal cavities filled with a $\mathrm{Cu}$-water nanofluid with an aiding or opposing side wall, Journal of Thermal Science and Engineering Applications, 8(3) (2016) 031009.

[14] F. Selimefendigil, H.F. Öztop, Modeling and optimization of MHD mixed convection in a lid-driven trapezoidal cavity filled with alumina-water nanofluid: effects of electrical conductivity models, International Journal of Mechanical Sciences, 136 (2018) 264-278.

[15] F. Selimefendigil, M.A. Ismael, A.J. Chamkha, Mixed convection in superposed nanofluid and porous layers in square enclosure with inner rotating cylinder, International Journal of Mechanical Sciences, 124 (2017) 95-108.

[16] E. Lewis, Steady flow between a rotating circular cylinder and fixed square cylinder, Journal of Fluid Mechanics, 95(3) (1979) 497-513.

[17] W.-S. Fu, C.-S. Cheng, W.-J. Shieh, Enhancement of natural convection heat transfer of an enclosure by a rotating circular cylinder, International Journal of Heat and Mass Transfer, 37(13) (1994) 18851897.

[18] T. Kimura, M. Takeuchi, K. Miyagawa, Effects of inner rotating horizontal cylinder on heat transfer in a differentially heated enclosure, Heat Transfer-Japanese Research, 24(6) (1995).

[19] A. Misirlioglu, The effect of rotating cylinder on the heat transfer in a square cavity filled with porous medium, International journal of engineering science, 44(18-19) (2006) 1173-1187.

[20] V. Costa, A. Raimundo, Steady mixed convection in a differentially heated square enclosure with an active rotating circular cylinder, International Journal of Heat and Mass Transfer, 53(5-6) (2010) 12081219.

[21] S.H. Hussain, A.K. Hussein, Mixed convection heat transfer in a differentially heated square enclosure with a conductive rotating circular cylinder at different vertical locations, International Communications in Heat and Mass Transfer, 38(2) (2011) 263-274.

[22] R. Roslan, H. Saleh, I. Hashim, Effect of rotating cylinder on heat transfer in a square enclosure filled with nanofluids, International Journal of Heat and Mass Transfer, 55(23-24) (2012) 7247-7256.

[23] M.H. Matin, I. Pop, Numerical study of mixed convection heat transfer of a nanofluid in an eccentric annulus, Numerical Heat Transfer, Part A: Applications, 65(1) (2014) 84-105.

[24] F. Selimefendigil, H.F. Öztop, MHD mixed convection of nanofluid filled partially heated triangular enclosure with a rotating adiabatic cylinder, Journal of the Taiwan Institute of Chemical Engineers, 45(5)
(2014) 2150-2162.

[25] C.-C. Liao, C.-A. Lin, Mixed convection of a heated rotating cylinder in a square enclosure, International Journal of Heat and Mass Transfer, 72 (2014) 9-22.

[26] Y.-C. Shih, Y.-J. Cheng, The effect of viscous dissipation on heat transfer in cavities of varying shape due to an inner rotating circular cylinder, Numerical Heat Transfer, Part A: Applications, 68(2) (2015) 150-173.

[27] F. Selimefendigil, H.F. Öztop, Mixed convection of nanofluids in a three dimensional cavity with two adiabatic inner rotating cylinders, International Journal of Heat and Mass Transfer, 117 (2018) 331-343.

[28] A.K. Hussein, H.K. Hamzah, F.H. Ali, L. Kolsi, Mixed convection in a trapezoidal enclosure filled with two layers of nanofluid and porous media with a rotating circular cylinder and a sinusoidal bottom wall, Journal of Thermal Analysis and Calorimetry, (2019) 1-19.

[29] Y. Hu, Y. He, C. Qi, B. Jiang, H.I. Schlaberg, Experimental and numerical study of natural convection in a square enclosure filled with nanofluid, International Journal of Heat and Mass Transfer, 78 (2014) 380-392.

[30] S. Giwa, M. Sharifpur, J. Meyer, Experimental study of thermoconvection performance of hybrid nanofluids of Al2O3MWCNT/water in a differentially heated square cavity, International Journal of Heat and Mass Transfer, 148 (2020) 119072.

[31] A.B. Solomon, J. van Rooyen, M. Rencken, M. Sharifpur, J.P. Meyer, Experimental study on the influence of the aspect ratio of square cavity on natural convection heat transfer with A12O3/Water nanofluids, International Communications in Heat and Mass Transfer, 88 (2017) 254-261.

[32] D. Wen, Y. Ding, Formulation of nanofluids for natural convective heat transfer applications, International Journal of Heat and Fluid Flow, 26(6) (2005) 855-864.

[33] P.S. Joshi, A. Pattamatta, Buoyancy induced convective heat transfer in particle, tubular and flake type of nanoparticle suspensions, International Journal of Thermal Sciences, 122 (2017) 1-11.

[34] E. Khalili, A. Saboonchi, M. Saghafian, Experimental study of nanoparticles distribution in natural convection of Al2O3-water nanofluid in a square cavity, International Journal of Thermal Sciences, 112 (2017) 82-91.

[35] F. Asdrubali, F. D'Alessandro, S. Schiavoni, A review of unconventional sustainable building insulation materials, Sustainable Materials and Technologies, 4 (2015) 1-17.

[36] K.H. Hilal Fluid Flow and Heat Transfer Characteristics in a Vertical Tube Packed Bed Media, University of Technology, (2004).

[37] P. Garg, J.L. Alvarado, C. Marsh, T.A. Carlson, D.A. Kessler, K. Annamalai, An experimental study on the effect of ultrasonication on viscosity and heat transfer performance of multi-wall carbon nanotubebased aqueous nanofluids, International Journal of Heat and Mass Transfer, 52(21-22) (2009) 5090-5101.

[38] B.C. Pak, Y.I. Cho, Hydrodynamic and heat transfer study of dispersed fluids with submicron metallic oxide particles, Experimental Heat Transfer an International Journal, 11(2) (1998) 151-170. 
$\omega$

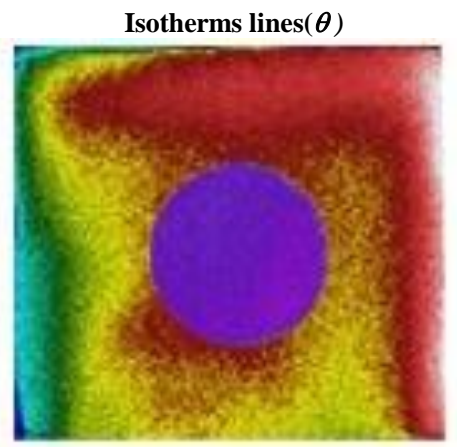

$-25$

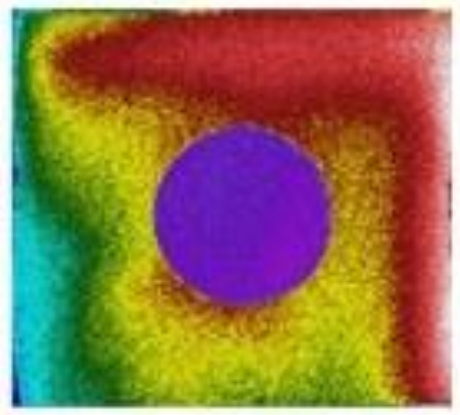

0

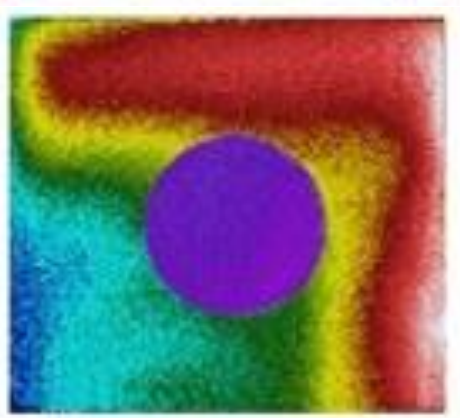

25

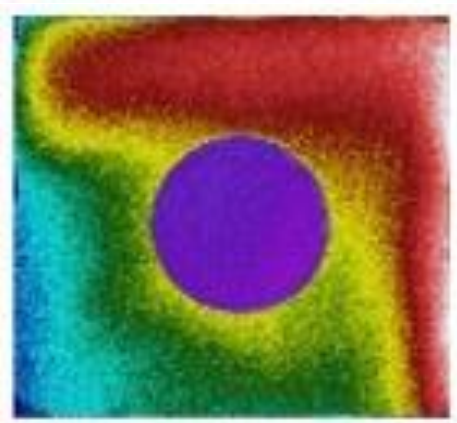

50

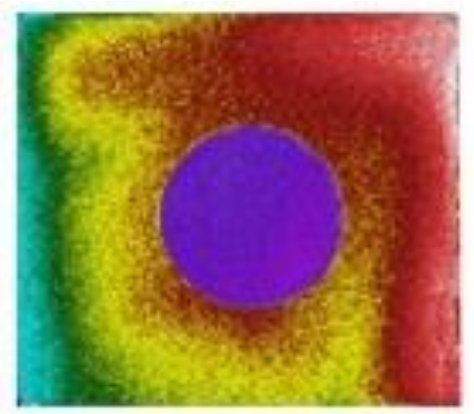

2IS

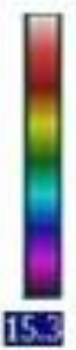

ERA

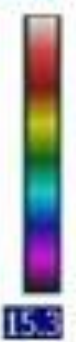

[2].

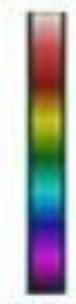

15.

E14

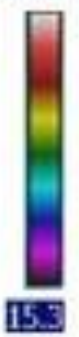

214.

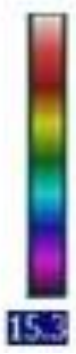

152
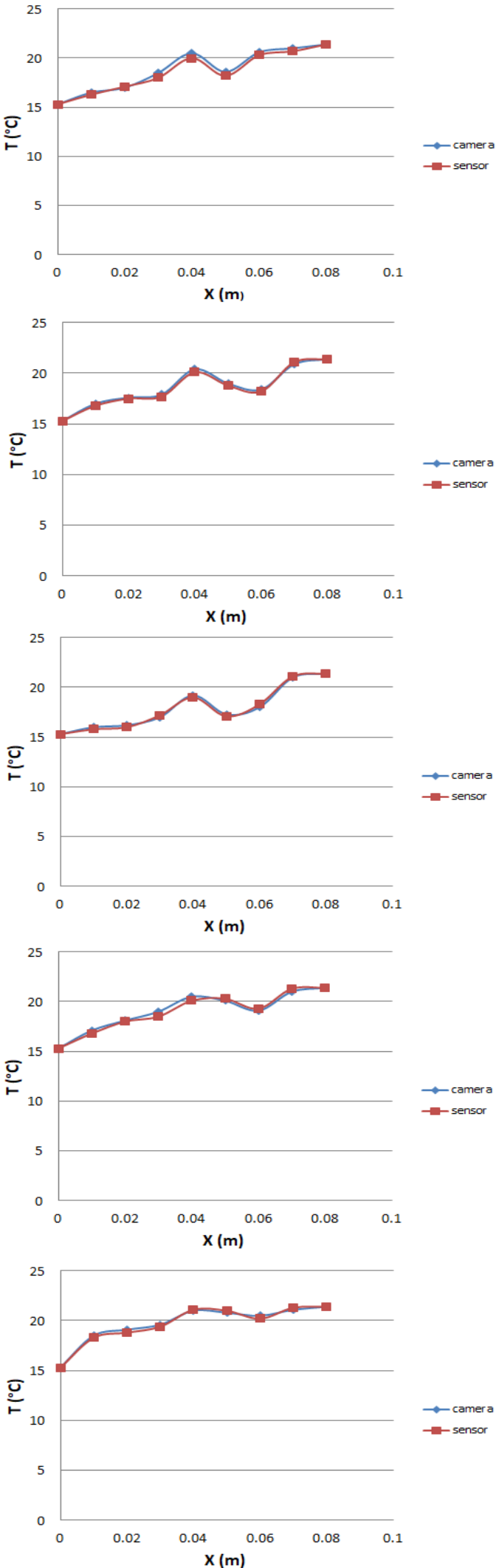

Figure 8. Temperature distribution in the enclosure at various Figure 9. Temperature distribution with $x$-axis at the middle line of the angular rotation velocity for $\Delta T=6$ enclosure at various angular rotation velocities for $\Delta T=6$ 
$\omega$

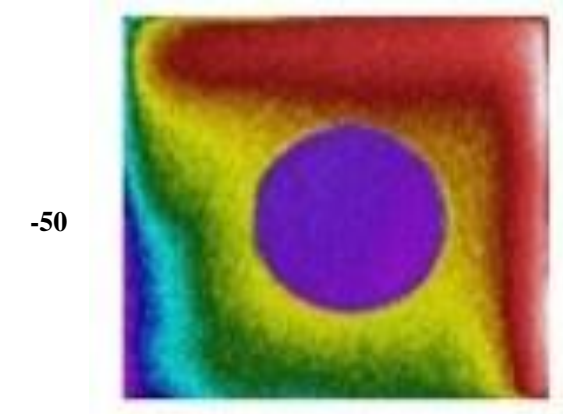

$-25$

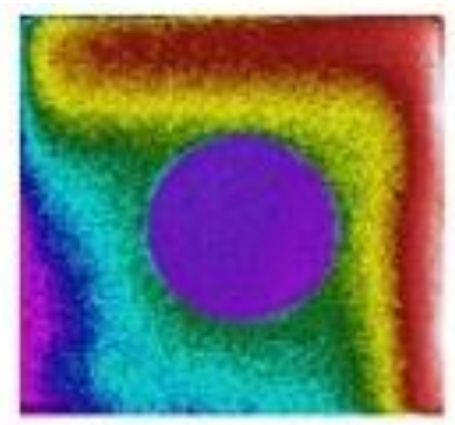

0

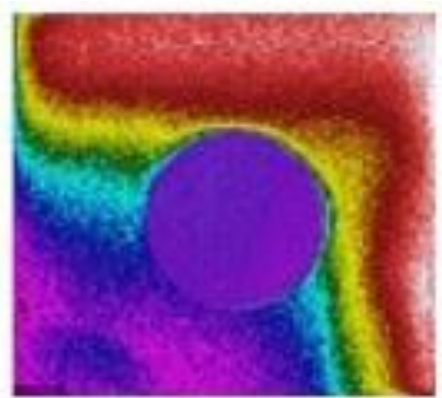

25

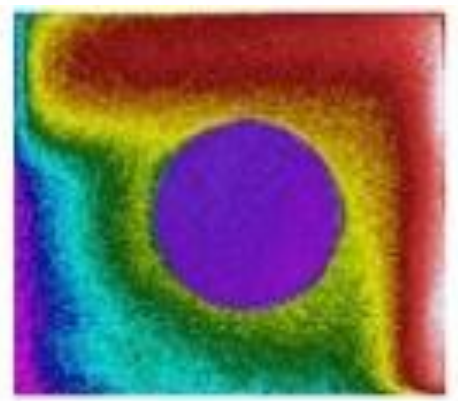

50

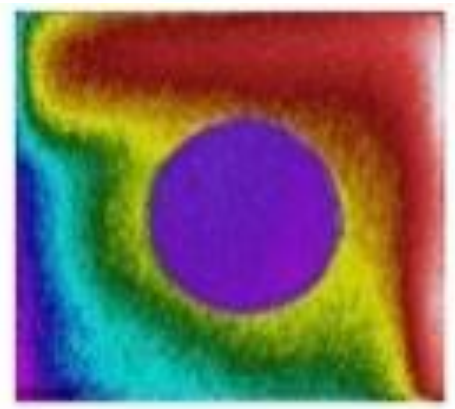

25]

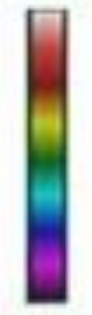

[․․]

20곤

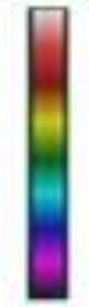

[5]

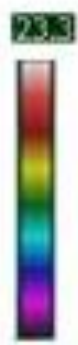

[5]

230

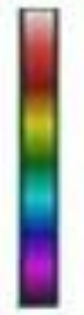

[15]
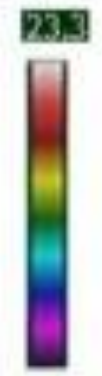

[15.1

Figure 10. Temperature distribution in the enclosure at various angular rotation velocity for $\Delta T=8$
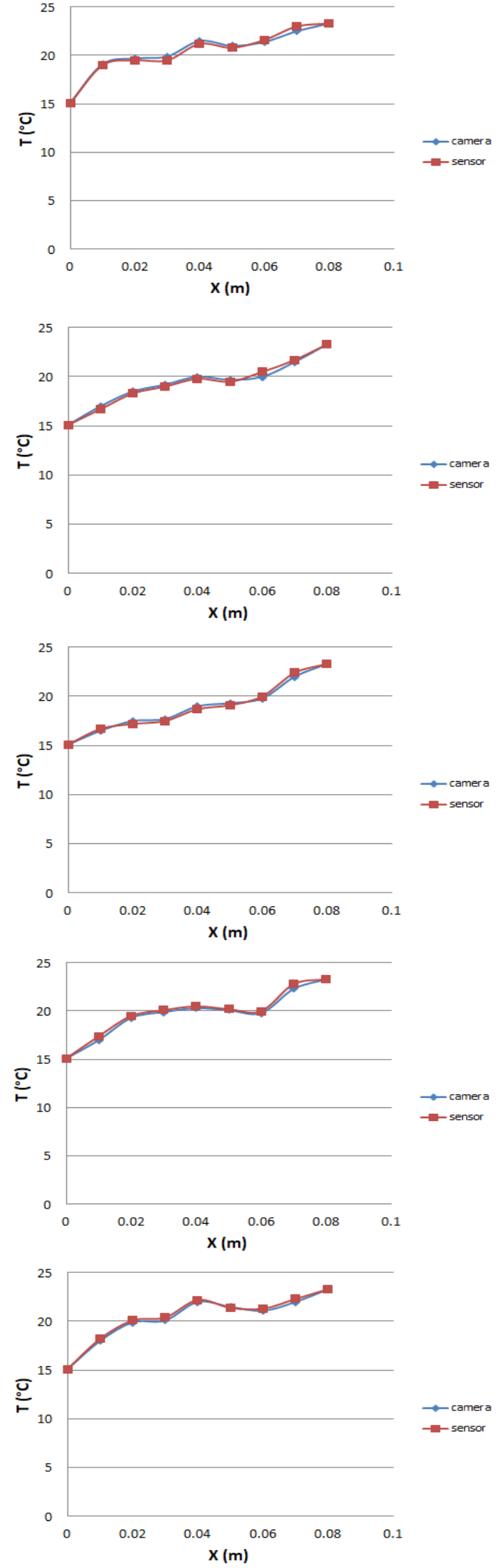

Figure 11. Temperature distribution with $x$-axis at the middle line of the enclosure at various angular rotation velocities for $\Delta T=8$ 
$\omega$

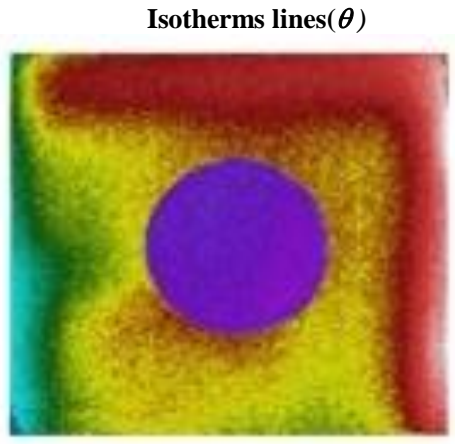

$-25$

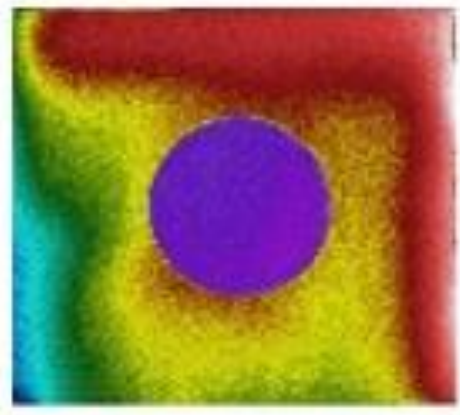

$\mathbf{0}$

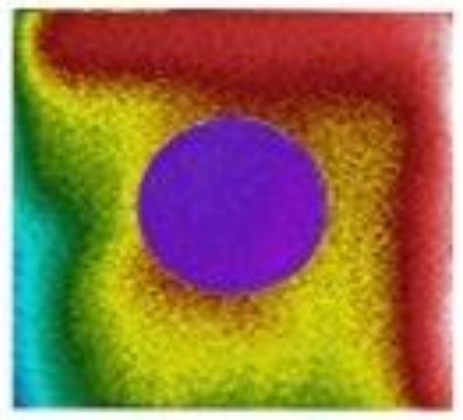

25

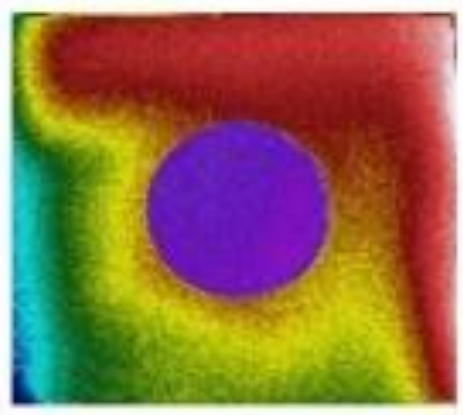

50

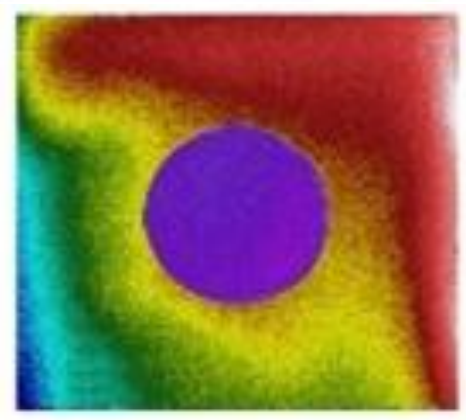

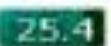

15.4

25.4

15.4

25.4

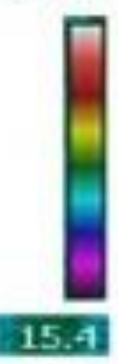

25.4

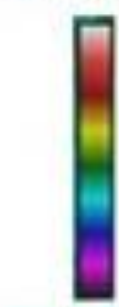

15.4
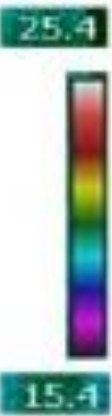
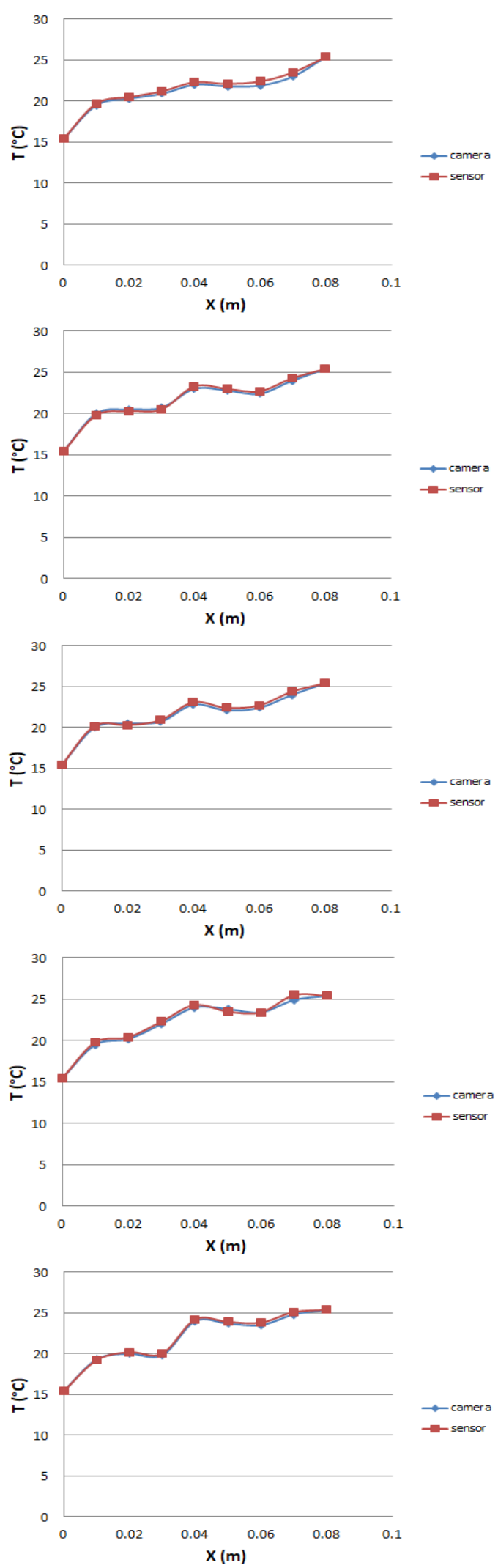

Figure 12. Temperature distribution in the enclosure at various Figure 13. Temperature distribution with $x$-axis at the middle line of the angular rotation velocity for $\Delta T=10$ enclosure at various angular rotation velocities for $\Delta T=10$ 